\title{
Characterization of Keto and Enol Forms of Indoline-2,3-dione-3-oxime using Avogadro software Tool (A Theoretical Study)
}

\author{
KATANGURU LAXMI \\ Department of Chemistry, Chaitanya Bharathi Institute of Technology (CBIT), \\ Gandipet, Hyderabad -500 075, India. \\ ${ }^{*}$ Corresponding author E-mail: katangurulaxmi@gmail.com \\ http://dx.doi.org/10.13005/ojc/340310
}

(Received: January 01, 2018; Accepted: March 09, 2018)

\begin{abstract}
Avogadro is a scientific software tool used as a molecule editor and visualizer. Avogadro is very much useful in teaching as it helps in the building of realistic models. Indoline-2,3-dione-3-oxime (IDOX) (Isatin-3-oxime) is used as a anticonvulsant and is known for treating various disorders caused by release of amino acids responsible for anxiety and excitatory behavior. In view of the biological importance of IDOX, the keto and enol forms of the molecule were constructed by using Avogadro 1.1 software, With the help of this software the molecular properties, atom Properties and bond properties of both forms of IDOX were determined and the presence of tautomerism between both forms of the compound IDOX is indicated. Standard molecular structure representations of keto form and enol forms of the compound IDOX so obtained by using chemical builder Avogadro 1.1 enabled to understand the structural aspects of both forms of IDOX.
\end{abstract}

Keywords: Avogadro, Anticonvulsant activity, Indoline-2,3-dione-3-oxime, Keto \& enol forms, Standard molecular structure representations.

\section{INTRODUCTION}

Avogadro is a well formed acceptable chemical builder which is very much helpful in visualization and analysis. Avogadro project is well designed molecule editor and visualizer with applications in computational chemistry, molecular modeling, bioinformatics, materials science, architecture and related areas. More refined version with additional features is
Avogadro version 1.1 which is applied even in crystallography.

The Avogadro project was introduced in 2007 and was downloaded over 270,000 times during the first 5 years of its development and was translated into over 20 languages. Avogadro project has over 20 contributors and has about 100 citations with respect to applications in various fields like theoretical chemistry, biochemistry, materials chemistry, spectroscopy and molecular dynamics'.

This is an Open Access article licensed under a Creative Commons Attribution-Non Commercial-Share Alike 4.0 International License (https://creativecommons.org/licenses/by-nc-sa/4.0/), which permits unrestricted Non Commercial use, distribution and reproduction in any medium, provided the original work is properly cited. 


\section{Anticonvulsant activity of Indoline-2,3-dione-3-oxime}

Neurotransmitters on increased excitation will lead to the death of neurons. Excitatory amino acids are also responsible to the degeneration of neurons in disorders such as cerebral ischemia or cerebral infarction leading to bad effects like hemorrhagic stroke, cardiac arrest and hypoglycemia. Compounds capable of blocking excitatory amino acid receptors have therapeutic use for the above breakdown effects. one such compound is Indoline-2,3-dione-3-oxime (Isatin-3-oxime).

Literature studies revealed that anticonvulsant ${ }^{2-4}$ screening of Indoline-2,3-dione-3oxime (IDOX)was performed by Maximal Electroshock (MES) model at dosage of 30, 100 and $300 \mathrm{mgkg}^{-1}$. IDOX was found to be active in the MES test at a lower dose of $100 \mathrm{mgkg}^{-1}$. This compound IDOX was also found to be more potent than standard drug sodium valproate. In view of biological importance of Indoline-2,3-dione-3-oxime (IDOX), in the present paper we report the structural aspects of IDOX in detail theoretically using Avogadro version 1.1.

\section{RESULTS AND DISCUSSIONS}

\section{Avogadro study of Indoline-2,3-dione-3-oxime (IDOX)} Avogadro ${ }^{5-10}$ is a "molecular editor," designed to be easy to use to construct and view molecules and materials in 3D. It runs on Windows, Linux, and Mac. It allows drawing molecules and also helps in creating a molecule. Molecules can be built and edited with the draw tool.

In Avogadro project by making use of various commands the information about molecule properties can be obtained. It is very much helpful to adjust even the Cartesian coordinates of the atoms in the molecule. Avogadro 1.1 support for naming compounds using the NIH Chemical Resolver system and the Pub Chem database. Initially Avogadro was designed with the use of Modern software design processes and also it has good agreement with other open-source projects which enables the use of all the practical code.

Some of these projects are Qt ${ }^{11}$ used as a graphical toolkit, Open Babel ${ }^{12}$ used for chemical file input/output and geometry optimization, Eigen ${ }^{13}$ useful for matrix and vector mathematics , OpenGL/ GLSL used for three-dimensional rendering.
Portable $\mathrm{C}++$ code is used for writing the core of Avogadro with some specifications formed by Qt, OpenGL, and Open Babel. Avogadro has been well framed and is well established on Linux, Apple Mac OS X, and Microsoft Windows in hardware architectures of common 32 and 64 bit.

\section{Building a molecule: atom by atom}

In the Avogadro window the draw tool is selected and a carbon atom is drawn by giving a left click on the display. Likewise by selecting the required atom from the periodic table the molecule is constructed. By the left clicking the bonds we can change the bond orders to single, double and triple as required to build the molecule.

If the bonds or atoms are right-clicked then they are deleted. By clicking the "Adjust Hydrogens" box or "Add hydrogens" option in the build menu the number of hydrogens on each atom are adjusted automatically so as to satisfy the valency accordingly.

The "atom centric manipulate" tool and the "bond centric manipulate" tool are the tools used for organizing the position of atoms in the molecules. By making use of "atom centric manipulate" tool it is possible to move an atom or a group of selected atoms. With the help of "bond centric manipulate" tool a bond is selected and the positions of all atoms relative to that bond is adjusted in different ways like modifying the bond length, bond angles, or dihedral angles.

Bond order can be changed through the Bond Order drop down menu, or by typing the numbers "1", "2", or "3". Bonds can also be added to a molecule by left clicking on a bond that has already been created (this process cycles through single, double, and triple bonds). Right clicking on a bond will delete the bond, and the atom it's bonded to.

As the building of molecular structure is completed, geometry optimization of the molecule is done by clicking on "Extensions" and "Optimize Geometry" buttons. Then adjustment of force field and calculation parameters is done.

In the present paper keto and enol forms of Indoline-2,3-dione-3-oxime(IDOX) (Fig.1)were built using Avogadro software and the existence of above 
forms in this molecule is proved by measuring the bond lengths ${ }^{14,15}$

The "Properties" selection will provides you with molecule, atom, bond, angle, torsion, and conformer properties. These settings display general

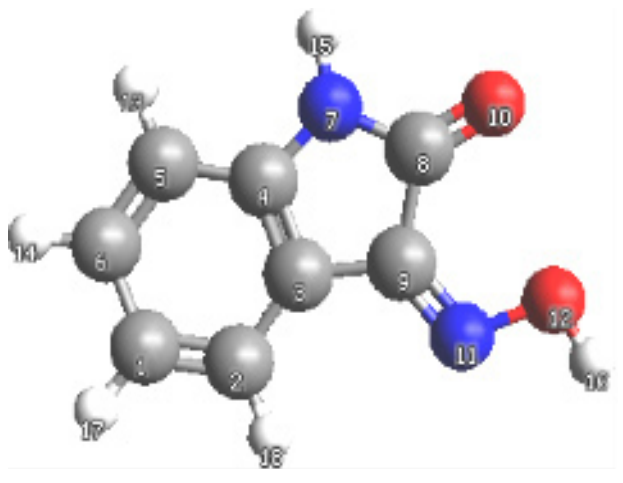

(i) keto form compositor information about the molecule and atoms present. For example, clicking on "Molecule Properties" will display general molecular information like molecular weight, chemical formulae energy $\mathrm{KJ} /$ mole , estimated dipole moment, number of atoms and bonds.

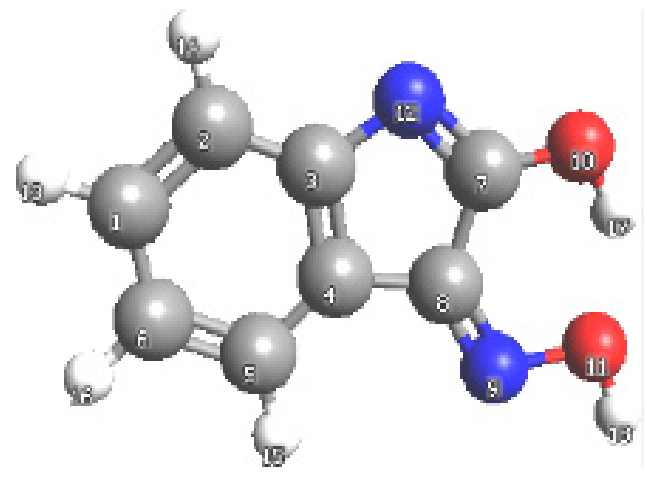

(ii) enol form

Fig. 1. Structure of keto \& enol forms of Indoline-2,3-dione-3-oxime(IDOX) as built by Avogadro ( ball \& stick with label )

Table 1: Molecule Properties of keto and enol forms of Indoline-2,3-dione-3-oxime(IDOX)

\begin{tabular}{lcc}
\hline Moleculer property & Keto form & Enol form \\
\hline Molecular Weight (g/mol) & 162.145 & 162.145 \\
Chemical Formulae & $\mathrm{C}_{8} \mathrm{H}_{6} \mathrm{~N}_{2} \mathrm{O}_{2}$ & $\mathrm{C}_{8} \mathrm{H}_{6} \mathrm{~N}_{2} \mathrm{O}_{2}$ \\
Energy KJ/ mole Conformer 1 & $329.8626 \mathrm{KJ} / \mathrm{mol}$ & $282.258 \mathrm{KJ} / \mathrm{mol}$ \\
Estimated Dipole Moment (D) & 3.819 & 3.119 \\
Number of atoms & 18 & 18 \\
Number of bonds & 19 & 19 \\
\hline
\end{tabular}

The molecular properties obtained keto and enol forms of IDOX are given in Table.1. The energy of keto and enol forms IDOX is $329.8626 \mathrm{KJ} / \mathrm{mol}$ and $282.258 \mathrm{KJ} / \mathrm{mol}$ respectively and it is endothermic .Dipole moment of keto and enol forms IDOX is 3.819 $\mathrm{D}$ and $3.119 \mathrm{D}$ respectively.

The atom properties of both keto and enol forms of IDOX were determined using Avogadro software. The valency, partial charge, formal charge of all elements of both forms are given in Table 2.

Mass spectrum recorded experimentally for the compound IDOX shows total peaks of 21 with Top Peak at $118 \mathrm{~m} / \mathrm{z}, 2^{\text {nd }}$ Highest peak at $91 \mathrm{~m} / \mathrm{z}$ and $3^{\text {rd }}$ Highest peak at $162 \mathrm{~m} / \mathrm{z}$.The molecular weight of Indoline-2,3-dione-3-oxime(IDOX) which is $162 \mathrm{~g} /$ $\mathrm{mol}$ is in agreement with the mass spectral data of the compound which shows $3^{\text {rd }}$ highest peak at 162 $\mathrm{m} / \mathrm{z}$. This confirms the existence of the compound IDOX in enolic form ${ }^{16}$.

keto-enol tautomerism in Indoline-2,3-dione-3oxime(IDOX)

To establish the existence of keto-enol tautomerism inIndoline-2,3-dione-3-oxime(IDOX) both keto and enol forms of Indoline-2,3-dione3-oxime(IDOX) were built by using Avogadro software(version 1.1) and the existence of keto and 
enol form in this molecule is proved by measuring the keto \& enol forms of Indoline-2,3-dione-3-oxime(IDOX) bond lengths. Atom Properties and Bond properties of were given in Tables 2 \& 3 respectively.

Table 2: Atom Properties of keto and enol forms of Indoline-2,3-dione-3-oxime(IDOX)

\begin{tabular}{|c|c|c|c|c|c|c|c|c|c|c|c|}
\hline \multicolumn{6}{|c|}{ Keto form of Indoline-2,3-dione-3-oxime (IDOX) } & \multicolumn{6}{|c|}{ Enol form of Indoline-2,3-dione-3-oxime (IDOX) } \\
\hline Atom & Type & Element & Valence & $\begin{array}{l}\text { Formal } \\
\text { Charge }\end{array}$ & $\begin{array}{l}\text { Partial } \\
\text { Charge }\end{array}$ & Atom & Type & Element & Valence & $\begin{array}{l}\text { Formal } \\
\text { Charge }\end{array}$ & $\begin{array}{l}\text { Partial } \\
\text { Charge }\end{array}$ \\
\hline 1 & $\mathrm{C} 2$ & $\mathrm{C} 1$ & 3 & 0 & -0.053 & 1 & Caro & $\mathrm{C} 1$ & 3 & 0 & -0.06 \\
\hline 2 & $\mathrm{C} 2$ & $\mathrm{C} 2$ & 3 & 0 & -0.016 & 2 & Caro & $\mathrm{C} 2$ & 3 & 0 & -0.036 \\
\hline 3 & $\mathrm{C} 1$ & C3 & 3 & -1 & -0.073 & 3 & Caro & C3 & 3 & 0 & 0.077 \\
\hline 4 & C1 & C4 & 3 & -1 & -0.057 & 4 & Caro & C4 & 3 & 0 & 0.034 \\
\hline 5 & $\mathrm{C} 2$ & C5 & 3 & 0 & -0.005 & 5 & Caro & C5 & 3 & 0 & -0.05 \\
\hline 6 & C2 & C6 & 3 & 0 & -0.052 & 6 & Caro & C6 & 3 & 0 & -0.061 \\
\hline 7 & Namine & N7 & 3 & 0 & -0.246 & 7 & C2 & C7 & 3 & 0 & 0.243 \\
\hline 8 & C2 & C8 & 3 & 0 & 0.284 & 8 & $\mathrm{C} 2$ & C8 & 3 & 0 & 0.173 \\
\hline 9 & $\mathrm{C} 2$ & $\mathrm{C} 9$ & 3 & 0 & 0.208 & 9 & N2 & N9 & 2 & 0 & -0.082 \\
\hline 10 & $\mathrm{O} 2$ & 010 & 1 & 0 & -0.267 & 10 & $\mathrm{O} 2$ & O10 & 2 & 0 & -0.491 \\
\hline 11 & $\mathrm{~N} 2$ & N11 & 2 & 0 & -0.074 & 11 & $\mathrm{O} 2$ & 011 & 2 & 0 & -0.409 \\
\hline 12 & $\mathrm{O} 2$ & 012 & 2 & 0 & -0.408 & 12 & N2 & N12 & 2 & 0 & -0.211 \\
\hline 13 & $\mathrm{HC}$ & $\mathrm{H} 13$ & 1 & 0 & 0.072 & 13 & $\mathrm{HC}$ & $\mathrm{H} 13$ & 1 & 0 & 0.062 \\
\hline 14 & $\mathrm{HC}$ & $\mathrm{H} 14$ & 1 & 0 & 0.062 & 14 & $\mathrm{HC}$ & $\mathrm{H} 14$ & 1 & 0 & 0.064 \\
\hline 15 & $\mathrm{HC}$ & $\mathrm{H} 15$ & 1 & 0 & 0.165 & 15 & $\mathrm{HC}$ & $\mathrm{H} 15$ & 1 & 0 & 0.062 \\
\hline 16 & $\mathrm{HO}$ & $\mathrm{H} 16$ & 1 & 0 & 0.328 & 16 & $\mathrm{HC}$ & $\mathrm{H} 16$ & 1 & 0 & 0.062 \\
\hline 17 & $\mathrm{HC}$ & $\mathrm{H} 17$ & 1 & 0 & 0.062 & 17 & $\mathrm{HO}$ & $\mathrm{H} 17$ & 1 & 0 & 0.294 \\
\hline 18 & $\mathrm{HC}$ & H18 & 1 & 0 & 0.071 & 18 & $\mathrm{HO}$ & $\mathrm{H} 18$ & 1 & 0 & 0.328 \\
\hline
\end{tabular}

Table 3: Bond properties of keto \& enol forms of Indoline-2,3-dione-3-oxime(IDOX)

\begin{tabular}{|c|c|c|c|c|c|c|c|c|c|c|c|}
\hline \multicolumn{7}{|c|}{ Keto form of IDOX } & \multicolumn{5}{|c|}{ Enol form of IDOX } \\
\hline Bond & $\begin{array}{l}\text { Start } \\
\text { atom }\end{array}$ & $\begin{array}{l}\text { End } \\
\text { atom }\end{array}$ & $\begin{array}{l}\text { Bond } \\
\text { order }\end{array}$ & Rotatable & $\begin{array}{l}\text { Length } \\
\left({ }^{\circ} \mathrm{A}\right)\end{array}$ & Bond & $\begin{array}{l}\text { Start } \\
\text { atom }\end{array}$ & $\begin{array}{l}\text { End } \\
\text { atom }\end{array}$ & $\begin{array}{l}\text { Bond } \\
\text { order }\end{array}$ & Rotatabl & $\begin{array}{l}\text { e Length } \\
\left({ }^{\circ} \mathrm{A}\right)\end{array}$ \\
\hline 1 & 5 & 4 & 1 & No & 1.441 & 1 & 1 & 2 & 2 & No & 1.4014 \\
\hline 2 & 1 & 2 & 2 & No & 1.3497 & 2 & 3 & 2 & 1 & No & 1.3814 \\
\hline 3 & 6 & 5 & 2 & No & 1.3501 & 3 & 6 & 1 & 1 & No & 1.409 \\
\hline 4 & 1 & 6 & 1 & No & 1.4658 & 4 & 5 & 6 & 2 & No & 1.3953 \\
\hline 5 & 4 & 3 & 3 & No & 1.3413 & 5 & 5 & 4 & 1 & No & 1.3801 \\
\hline 6 & 4 & 7 & 1 & No & 1.3573 & 6 & 3 & 12 & 1 & No & 1.4136 \\
\hline 7 & 7 & 8 & 1 & No & 1.3777 & 7 & 8 & 4 & 1 & No & 1.4482 \\
\hline 8 & 2 & 3 & 1 & No & 1.4363 & 8 & 8 & 7 & 1 & No & 1.5131 \\
\hline 9 & 8 & 10 & 2 & No & 1.2097 & 9 & 3 & 4 & 2 & No & 1.3983 \\
\hline 10 & 9 & 8 & 1 & No & 1.5471 & 10 & 12 & 7 & 2 & No & 1.2971 \\
\hline 11 & 3 & 9 & 1 & No & 1.4738 & 11 & 7 & 10 & 1 & No & 1.3196 \\
\hline 12 & 11 & 9 & 2 & No & 1.2949 & 12 & 9 & 8 & 2 & No & 1.2947 \\
\hline 13 & 11 & 12 & 1 & No & 1.396 & 13 & 11 & 9 & 1 & No & 1.3985 \\
\hline 14 & 5 & 13 & 1 & No & 1.0833 & 14 & 1 & 13 & 1 & No & 1.0893 \\
\hline 15 & 6 & 14 & 1 & No & 1.0856 & 15 & 2 & 14 & 1 & No & 1.0853 \\
\hline 16 & 7 & 15 & 1 & No & 1.0086 & 16 & 5 & 15 & 1 & No & 1.0846 \\
\hline 17 & 12 & 16 & 1 & No & 0.9745 & 17 & 6 & 16 & 1 & No & 1.0886 \\
\hline 18 & 1 & 17 & 1 & No & 1.0855 & 18 & 10 & 17 & 1 & No & 0.9758 \\
\hline 19 & 2 & 18 & 1 & No & 1.0839 & 19 & 11 & 18 & 1 & No & 0.9792 \\
\hline
\end{tabular}


Avogrado software tool allows to determine bond lengths, angles, and dihedrals. With the help of measure tool it is possible to select and assess up to four atoms. As you click on atoms Avogadro will automatically calculate the distances between atoms in a respective order. When three atoms are selected an angle is measured between the first and third atom, and the second atom is the vertex. The align tool rotates, and translates a molecule(s) into a specific reference frame. An alignment axis can be chosen from the "Axis" drop down menu. Typing "x", "y", or "z" is a shortcut for changing the alignment axis.

Table 4: Comparitive study of bond lengths of keto \& enol forms of Indoline-2,3-dione-3-oxime(IDOX)

$\begin{array}{rrrrrrr}\text { IDOX(keto) Start atom } & \text { End atom } & \left.\text { Bond order Length ( }{ }^{\circ} \mathrm{A}\right) & \begin{array}{r}\text { Type of } \\ \text { Bond }\end{array} \\ \text { Bond } 7 & 7 & 8 & 1 & 1.3777 & \mathrm{~N}-\mathrm{C} \\ \text { Bond } 9 & 8 & 10 & 2 & 1.2097 & \mathrm{C}=\mathrm{O} \\ \text { Bond } 16 & 7 & 15 & 1 & 1.0086 & \mathrm{~N}-\mathrm{H}\end{array}$

$\begin{array}{cccccccc}\text { IDOX(enol) Start atom } & \text { End atom } & \text { Bond order } & \begin{array}{c}\text { Length } \\ \left({ }^{\circ} \mathrm{A}\right)\end{array} & \begin{array}{c}\text { Bond } \\$\cline { 3 - 7 }\end{array} \\ \text {$Bond 10 } & 12 & 7 & 2 & 1.2971 & \mathrm{~N}=\mathrm{C} \\ \text { Bond 11 } & 7 & 10 & 1 & 1.3196 & \mathrm{C}-\mathrm{O} \\ \text { Bond 18 } & 10 & 17 & 1 & 0.9758 & \mathrm{O}-\mathrm{H}\end{array}$

A Comparitive study of bond lengths of keto \& enol forms of Indoline-2,3-dione-3-oxime(IDOX) is given in Table 4. In Avogrado study of IDOX the keto form shows bond between atoms 8 and 1 indicating $\mathrm{N}-\mathrm{C}$ bond with bond length of $1.3777\left({ }^{\circ} \mathrm{A}\right)$. This is comparable to the bond between atoms 12 and 7 of enol form indicating $\mathrm{N}=\mathrm{C}$ in enol form with bond length of $1.2971\left({ }^{\circ} \mathrm{A}\right)$. The decrease in the bond length so observed is an evidence for manifestation of keto- enol tautomerism in IDOX.

The existence of tautomerism between the keto and enol forms of the compound IDOX is confirmed by the following factors

i. In enol form of IDOX the bond length of 1.3196 ${ }^{\circ} \mathrm{A}$ corresponding to $\mathrm{C}-\mathrm{O}$ is observed. But in keto form of IDOX the bond length of 1.2097 ${ }^{\circ} \mathrm{A}$ between the same atoms is indicative of $\mathrm{C}=\mathrm{O}$ bond. This is further confirming the existence of keto-enol tautomerism in IDOX.

ii. Further the enol form of IDOX shows $\mathrm{O}-\mathrm{H}$ bond having the bond length of $0.9758^{\circ} \mathrm{A}$. The absence of this bond in keto form is indicative proof of keto-enol tautomerism in IDOX.

iii. However the subsisitence of tautomerism between the keto and enol forms of the compound IDOX, is in agreement with the IR and ${ }^{1} \mathrm{H}$ NMR spectral data of IDOX recorded experimentally. ${ }^{1} \mathrm{H}$ NMR spectrum ${ }^{16}$ of IDOX at $24{ }^{\circ} \mathrm{C}$ in $\mathrm{CDCl}_{3}+3$ drops of DMSO-d6 shows peaks at $\delta 13.2 \mathrm{ppm}\left({ }^{1} \mathrm{H}\right.$, enolic $\left.-\mathrm{OH}\right)$, $\delta$ 10.59ppm $(1 \mathrm{H},=\mathrm{N}-\mathrm{OH})$ and $\delta 7.30-8.47$ $(4 \mathrm{H}, \mathrm{Ar}-\mathrm{H})$. In IR spectrum ${ }^{16}$ of IDOX recorded experimentally the peaks appeared at 3180 $\mathrm{cm}^{-1}$ (v enolicN-OH), $3050 \mathrm{~cm}^{-1}$ (v amineN-H), $2896 \mathrm{~cm}^{-1}$ (v aromaticC-H), $1713 \mathrm{~cm}^{-1}$ (v ketonic $\mathrm{C}=\mathrm{O}$ ).

\section{Standard representations of keto and enol forms of Indoline-2,3-dione-3-oxime(IDOX)}

Avogadro is a molecular editor used widely in computational chemistry, bioinformatics and related areas. Avogadro software tool is provided with various engine plugins like Ball and Stick display, Dipole moment display if present, Hydrogen Bond 
display, Overlay properties display, Polygon display, Ring display, Simple Wireframe display, Van der Waals Surface display, Van der Waals sphere display and Wireframe display
Several representations applied for keto and enol forms of Indoline-2,3-dione-3-oxime (IDOX) are given in Table 5. All these forms of representations are helpful in elucidating the molecule backbone.

Table 5: List of display type (engine) plugins in Avogrado applied for keto and enol forms of Indoline-2,3-dione-3-oxime (IDOX)

\begin{tabular}{|c|c|}
\hline display type & Representation of rendering of $\mathrm{x}, \mathrm{y}, \mathrm{z}$ Cartesian axes from the origin \\
\hline Ball and Stick display & Swaps Standard ball and stick representation \\
\hline Dipole moment display if present & restores direction/magnitude of dipole \\
\hline Hydrogen Bond display & restores hydrogen bonds as dotted lines \\
\hline Overlay properties display & Overlay of color gradient used for electrostatic \\
\hline Polygon display & restores closed polygons of metallic centers \\
\hline Ring display & restores rings in structure, different colors depending on ring size \\
\hline Simple Wireframe display & $\begin{array}{l}\text { Very simple wireframe display Sticks Stick or liquorices rendering } \\
\text { style for atoms and bonds }\end{array}$ \\
\hline Van der Waals Surface display & restores triangular isosurface meshes \\
\hline Van der Waals sphere display & $\begin{array}{l}\text { restores Vander Waals sphere rendering (no bonds, Spheres } \\
\text { space-filling) }\end{array}$ \\
\hline Wireframe display & Wireframe swaping more features such as bond order \\
\hline
\end{tabular}

Standard molecular structure representations of keto and enol forms of Indoline-2,

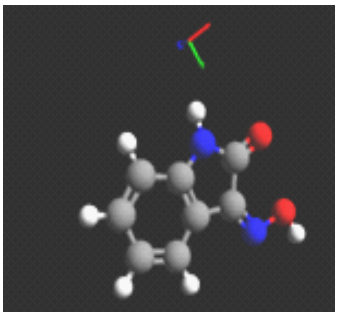

(a) ball and stick

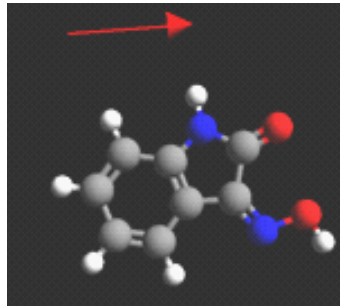

(b) dipole representation

3-Dione-3-Oxime (IDOX) are given in Figs 3 and 4 respectively.

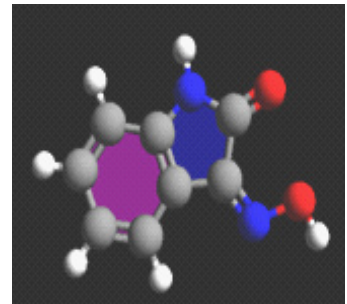

(c) ring representation

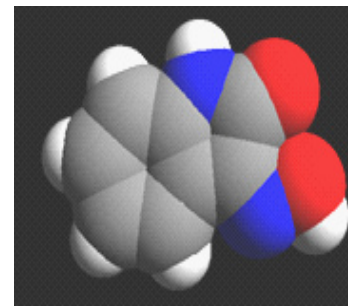

(d) Van der Waals spheres

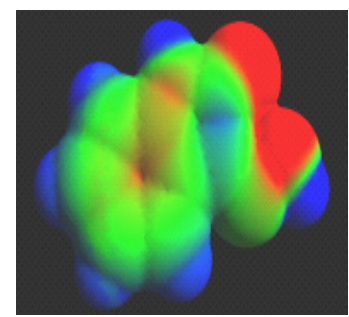

(e) Van der Waals surfaces (colored by electrostatic potential)

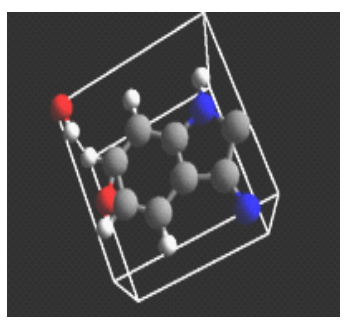

(f) unit cell

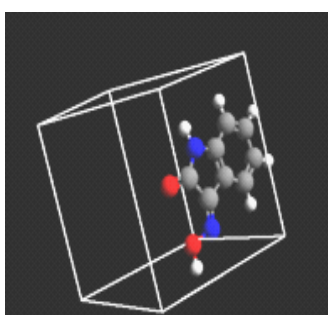

(g) super cell builder

Fig. 2. Standard molecular structure representations of keto form of Indoline-2,3-dione-3-oxime a) ball and stick (b) dipole c) ring (d) Van der Waals spheres (e) Van der Waals surface (f) unit cell (g) super cell builder

Ball and Stick is the default plug-in when Avogadro is opened. This plug-in provides the standard ball and stick representation of a molecule.
The Dipole plug-in will display an overall net dipole if one is present. The label plug-in numbers and labels all atoms present in a molecule. The Force plug-in 
displays green arrows on atoms (as shown below), to qualitatively demonstrate the forces being applied to the atoms.

The Hydrogen Bond plug in demonstrates implicit hydrogen bonding that can occur between atoms. This plug-in is possible only for the enol form of IDOX (Figure 3).

The Polygon feature takes metallic centers with three or more atoms bonded to them, and draws a polygon around them. This feature distinguishes rings with different colors dependent on their size. As shown below a six-membered ring is purple, and five membered ring is blue, etc.

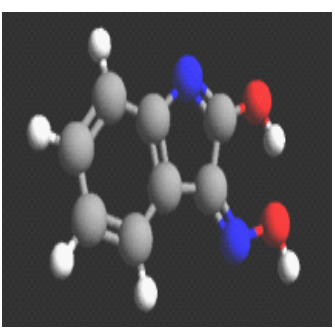

(a) ball and stick

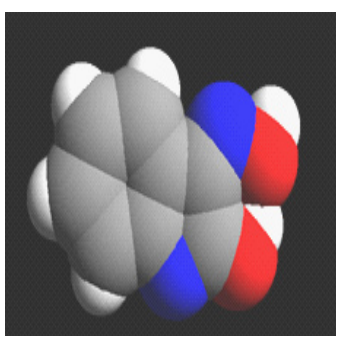

(e)Van der Waals spheres

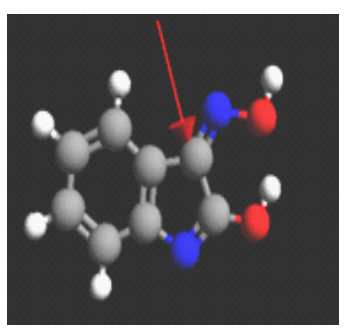

(b) dipole representation

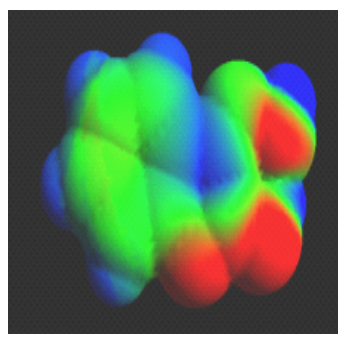

(f) Van der Waals surfaces

Once a surface has been created (Extensions Menu - > Create Surfaces...), the Surface display type can be used. This display type allows adjustments to the orbital, opacity, rendering, style, and color. The Van der Waals plug-in provides the classic sphere rendered Van der Waals image.

The electrostatic potential maps help to visualize charge distribution, and other charge related properties of molecules. When an electrostatic surface has been created, from this surface, one can interpret where the most electron density resides (in the more red areas), and where the least electron density resides (deep blue areas). You can further determine, and compare the acidity of various protons, and how surrounding atoms impact the overall electron density.

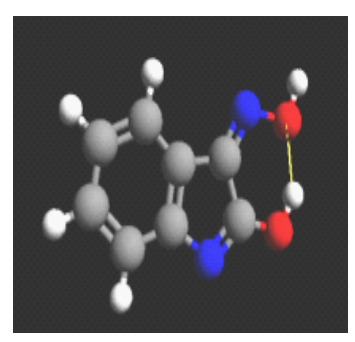

(c) $\mathrm{H}$ bond representation

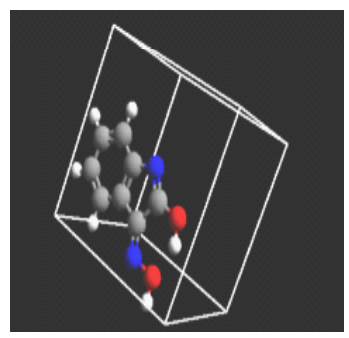

(g) unit cell

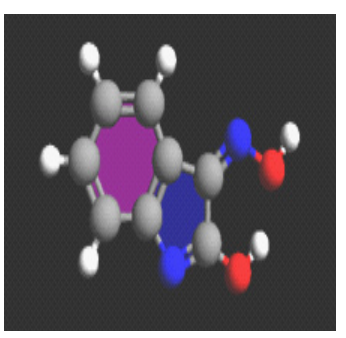

(d) ring representation

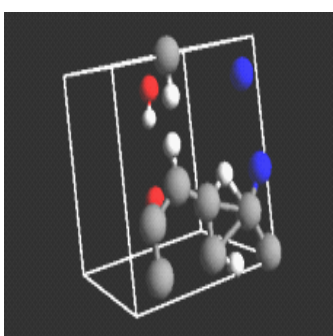

(h) super cell builder

Fig. 3. Standard molecular structure representations of enol form of Indoline-2,3-dione-3-oxime (a) ball and stick (b) dipole (c) H bond (d) ring (e) Van der Waals sphere (f)Van der Waals surface (g) unit cell(h) super cell builder

\section{CONCLUSION}

Avogadro study on the Indoline-2,3dione-3-oxime (IDOX) compound is informative in understanding various physicochemical aspects like molecule properties, atom properties, bond properties of the compound. These revealed the subsistence of both keto form and enol forms in the compound IDOX, between which there exists tautomerism. Standard molecular structure representations of keto and enol forms of Indoline-2,3-dione-3-oxime (IDOX) compound is very helpful in understanding the structural aspects of the compound.

\section{REFERENCES}

1. Mera-Adasme R, Mendiz'abal F, OleaAzar C, Miranda-Rojas S, Fuentealba P: A Computationally Efficient and Reliable Bond
Order Measure. J Phys Chem A., 2011, 115(17):4397-4405.

2. Smitha. S, Pandeya. S. N., Sci. Pharm., 2008, 76,621 . 
3. Pandeya. S. N, Kumar. R, Asia J. Res. Chem., 2010, 3(3): 646.

4. Pandeya S. N, IJPI's Journal of Drug Chemistry., 2012 , 2, 8,1-7.

5. Avogadro Downloads 2012. [http:// sourceforge.net/projects/avogadro/files/stats/ timeline?dates=2006-04-14+to+2012-03-29]

6. Avogadro Translations 2012. [https:// translations.launchpad.net/avogadro/ trunk/+translations].

7. Avogadro Contributors 2012. [http://www. ohloh.net/p/avogadro/ contributors].

8. Avogadro Citations from Google Scholar 2012. [http://scholar.google. com/scholar? \&amp;q=22http://avogadro.openmolecules. net/22].

9. Hanwell, Marcus D; Curtis, Donald E; Lonie, David C; Vandermeersch, Tim; Zurek, Eva; Hutchison, Geoffrey R. "Avogadro: An advanced semantic chemical editor, visualization, and analysis platform". J. Cheminform., 2012, 4 (1), 17. PMC 3542060, PMID 22889332. doi:10.1186/17582946-4-17.

10. http://avogadro.openmolecules.net/.

11. Qt Framework 2012. [http://qt-project.org/].
12. O'Boyle NM, Banck M, James CA, Morley C, Vandermeersch T, Hutchison GR: Open Babel: an open chemical toolbox. J Cheminf ., 2011, 3, 33.

13. Guennebaud G, Jacob B, Eigen v2. 2010. [http://eigen.tuxfamily.org].

14. RalMera-Adasme; Fernando Mendizbal; Claudio Olea-Azar; Sebastin Miranda-Rojas; Patricio Fuentealba. "A Computationally Efficient and Reliable Bond Order Measure". J. Phys. Chem., 2011, 115 (17): 4397-4405. PMID 21469689. doi:10.1021/jp107498h.

15. Michael Salciccioli; Weiting Yu; Mark A. Barteau; Jingguang G. Chen; Dionisios G. Vlachos "Differentiation of $\mathrm{O}-\mathrm{H}$ and $\mathrm{C}-\mathrm{H}$ Bond Scission Mechanisms of Ethylene Glycol on Pt and Ni/Pt Using Theory and Isotopic Labeling Experiments". J. Am. Chem. Soc., 2011,133(20), 7996-8004. PMID 21526776. doi:10.1021/ja201801t.

16. A study on structural aspects of indoline-2, 3-dione-3-oxime: Experimental and theoretical approach, K. Laxmi, International Journal of Computational and Theoretical Chemistry 2013, 1(2), 11-17. 